\title{
Endometrial stem cells repair injured endometrium and induce angiogenesis via AKT and ERK pathways
}

\author{
Yanling Zhang*, Xiaona Lin*, Yongdong Dai, Xiaoxiao Hu, Haiyan Zhu, Yinshen Jiang and \\ Songying Zhang \\ Department of Gynecology and Obstetrics, Sir Run Run Shaw Hospital, School of Medicine, Zhejiang University, \\ Hangzhou, Zhejiang Province, China \\ Correspondence should be addressed to S Zhang; Email: zhangsongying@126.com
}

*(Y Zhang and $\mathrm{X}$ Lin contributed equally to this work)

\begin{abstract}
Intrauterine adhesions are common acquired endometrial syndromes secondary to endometrial injury, with limited effective therapies. Recently, several studies have reported that bone marrow stem cells (BMSCs) could repair injured endometrium in animal experiments. However, the role of stem cells in endometrial injury repair and its therapeutic mechanisms remain unclear. Here, we established mouse endometrial injury model and examined the benefit of human endometrial mesenchymal stem cells derived from menstrual blood (MenSCs) in restoration of injured endometrium. Injured endometrium exhibited significantly accelerated restoration at Day 7 after MenSCs transplantation, with increased endometrial thickness and microvessel density. Moreover, the fertility of mice with injured endometrium was improved, with higher conception rate $(53.57 \%$ vs $14.29 \%, P=0.014)$ and larger embryo number $(3.1 \pm 0.6$ vs $0.9 \pm 0.7, P=0.030)$ in MenSCs group than control group, while no difference was found in undamaged horns between two groups. Conditioned medium from MenSCs (MenSCs-CM) could decrease $\mathrm{H}_{2} \mathrm{O}_{2}$-induced apoptosis of human umbilical vein endothelial cells (HUVECs) and promote proliferation, migration and angiogenesis. Angiogenesis effect of MenSCs-CM was also confirmed in Matrigel plug assay in mice. Furthermore, we discovered that MenSCs-CM could activate AKT and ERK pathways and induce the overexpression of eNOS, VEGFA, VEGFR1, VEGFR2 and TIE2 in HUVECs, which are critical in MenSCs-CM-induced angiogenesis. Angiogenesis induced by MenSCs-CM could be reversed by inhibitors of AKT and/or ERK. Taken together, we concluded that MenSCs could restore injured endometrium and improve the fertility of the endometrial injury mice, which was partially attributed to angiogenesis induced by MenSCs.

Reproduction (2016) 152 389-402
\end{abstract}

\section{Background}

Intrauterine adhesions (IUAs) are common acquired endometrial disorders secondary to endometrial injury such as trauma, curettage, infection, uterine congenital anomaly and genetic predisposition (Yu et al. 2008, March 2011). The incidence of IUAs has increased in recent years and $90 \%$ of IUAs are associated with damage to the uteri due to intrauterine surgery (Schenker \& Margalioth 1982). The leading pathological feature of IUAs is avascular fibrous connective tissue bands with or without glandular tissue. IUAs can reveal itself in a wide spectrum of clinical presentations, including a decrease in menstrual flow, infertility, recurrent miscarriage, amenorrhea and placenta accreta, all of which significantly influence the reproductive health of women (Yu et al. 2008).

Recently, transcervical resection of adhesions (TCRA) has been a conventional and standardized method of stripping adhesions and restoring the shape of uterine cavity with the development of hysteroscopy technology (Yu et al. 2008). However, postoperative endometrial restoration and prevention of recurrent IUAs are two challenges in clinical practice. At present, placement of intrauterine device (IUD) and application of artificial hormone therapy with high doses of estrogen are used in combination to prevent postoperative recurrence of IUAs and promote endometrial restoration and regeneration (Romer et al. 2000, Yu et al. 2008, Gargett \& Healy 2011). However, the modest efficacy of estrogen therapy, which is usually accompanied by adverse effects, and the inefficiency of IUD impel us to search for alternative therapeutic methods for IUAs.

Stem cell therapy is a promising method for incurable diseases and conditions. Paracrine actions have been considered as the predominant mechanism for the role of mesenchymal stem cells (MSCs) in tissue repair (Kinnaird et al. 2004b, Togel et al. 2007, Camussi et al. 2010). It has been proposed 
that condition medium from MSCs was effective for reducing myocardial ischemia (Jiang et al. 2013), improving limb reperfusion (Kinnaird et al. 2004b, Kwon et al. 2014) and protecting against acute kidney injury (Togel et al. 2007, Camussi et al. 2010). Recently, several studies have reported that bone marrow stem cells (BMSCs) could induce the regeneration of thin endometrium and injured endometrium in animal experiments (Alawadhi et al. 2014, Jing et al. 2014, Kilic et al. 2014). However, the potential function and the underlying molecular mechanism of MSCs in IUAs repair yet need to be uncovered.

Therapeutic angiogenesis by stem cell-based approaches has received increasing attention because stem cells could secrete many angiogenesisenhancing cytokines and growth factors (Boomsma \& Geenen 2012, Rahbarghazi et al. 2013, Kwon et al. 2014). Human endometrial mesenchymal stem cells derived from menstrual blood (MenSCs) have emerged as attractive, novel adult stem cells that are easily obtained and have demonstrated a potential capacity for clinical therapy. Superior angiogenetic properties of MenSCs in comparison to bone marrow stem cells (BMSCs) were observed in vitro and in Matrigel plug assays in mice (Alcayaga-Miranda et al. 2015). Preclinical studies have confirmed the beneficial effects of MenSCs on heart failure and critical limb ischemia (Murphy et al. 2008, Bockeria et al. 2013, Jiang et al. 2013). What is more, a recent study showed that the endometrial tissues in patients with IUAs presented with vascular closure and VEGF expression, and angiogenesis would be present in the endometrial tissues after treatment, indicating that angiogenesis in the injured endometrium may affect endometrial regeneration (Chen et al. 2013). In the light of the development of MenSCs as therapeutic strategy, we investigated whether transplantation of MenSCs induced the repair and angiogenesis of injured endometrium and explored the underlying mechanisms responsible for this improvement.

\section{Materials and methods}

\section{Animals}

ICR female and male mice were obtained from the Experimental Animal Center of Zhejiang Province and maintained in the Animal Facility of Sir Run Run Shaw Hospital, Zhejiang University. Five to six mice

Table 1 Pregnancy rate of damaged uterine horns in MenSCs and control groups.

\begin{tabular}{lccc}
\hline & Pregnancy & Nonpregnancy & P value \\
\hline MenSCs group & 15 & 13 & 0.014 \\
Control group & 2 & 12 & \\
\hline
\end{tabular}

The $\chi^{2}$ test was applied. $P<0.05$ was considered statistically significant. per cage were housed in a room with a $12 \mathrm{~h}$ light: $12 \mathrm{~h}$ darkness cycle and provided access to food and water ad libitum. All animals were treated according to a protocol approved by the Institutional Animal Care and Use Committee of Zhejiang University. Animal handlers were blinded to the experimental groups. Tissues obtained were also examined in a blinded fashion.

\section{Establishment of the mouse endometrial injury model by electrocoagulation}

ICR female mice, 6-7 weeks old, were used to establish the endometrial injury model by electrocoagulation as described previously (Hu et al. 2014). Briefly, after administration of $8 \%$ chloral hydrate $(0.1 \mathrm{~mL} / 10 \mathrm{~g})$ by intraperitoneal injection, a vertical incision was made in the abdominal wall and the uteri were exposed. A small incision was made in the right uterine horn, and then a copper wire of $1 \mathrm{~mm}$ in diameter (only the top of the wire was conductive, Supplementary Fig. 1A, see section on supplementary data given at the end of this article) was inserted into the lumen of the right uterine horn of each mouse through this incision. Electric coagulation was implemented to induce endometrial injury with $0.5 \mathrm{~W}$ of power whilst pressing the blue button and moving the wire towards the outside of the lumen (Supplementary Fig. 1A and B). The entire process took $3 \mathrm{~s}$. The left horns remained untreated as self-controls. The uteri were disinfected and then reset.

\section{MenSCs culture and labeling}

MenSCs were isolated and provided by the State Key Laboratory for Diagnosis and Treatment of Infectious Diseases, First Affiliated Hospital, School of Medicine, Zhejiang University (Mou et al. 2013, Wu et al. 2014, Lai et al. 2015). Briefly, human menstrual blood was collected

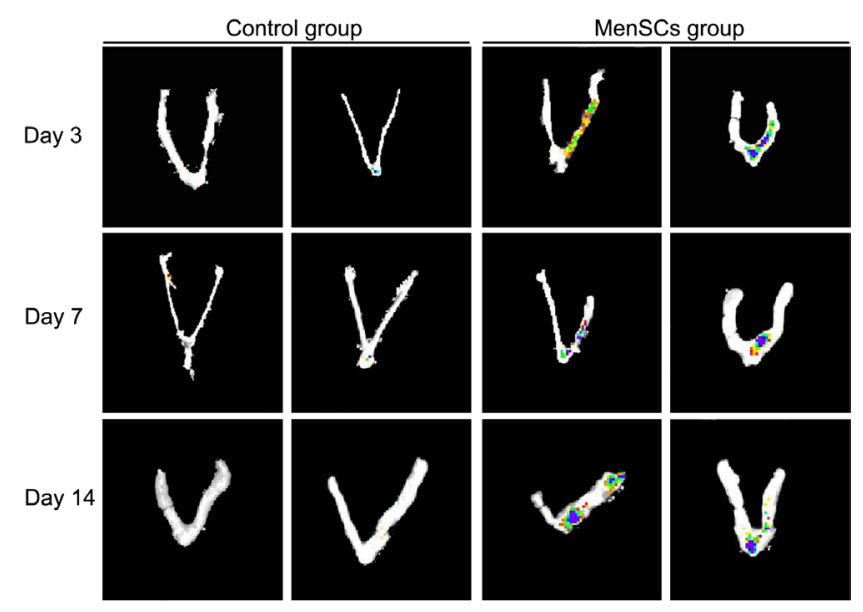

Figure 1 Dil-labeled MenSCs are recruited to the endometrium in response to injury. After the right uteri were injured by electrocoagulation, MenSCs or normal saline were injected into the endometrial injury mice via tail vein injection at Day 3 after injury. Fluorescent signals were captured by in vivo living imaging system (IVIS) at Day 3, Day 7 and Day 14 post transplantation ( $n=3$ per group at each time point). 
using a DivaCup (E-vans Biotech, Hangzhou, China) during the first day of menstruation. The cells were transferred into phosphate-buffered saline (PBS) with mixed antibiotics, including amphotericin B, gentamycin sulfate, kanamycin sulfate, cephalexin, vancomycin hydrochloride and heparin at $4{ }^{\circ} \mathrm{C}$ for $24 \mathrm{~h}$. Then, the samples were centrifuged at $1600 \mathrm{~g}$ for $10 \mathrm{~min}$ at $4{ }^{\circ} \mathrm{C}$, and the supernatants were used for microbiological testing. Mononuclear cells were separated by Ficoll-Paque $(1.077 \mathrm{~g} / \mathrm{mL}$, Thermo Fisher Scientific) density-gradient centrifugation according to the manufacturer's instructions. The purified mononuclear cells were cultured in the Chang Medium (Patel et al. 2008) overnight at $37^{\circ} \mathrm{C}$ in $5 \% \mathrm{CO}_{2}$. Cells were trypsinized, subcultured and passaged every 4-6 days. Cells were used for experiments until they reached $80-90 \%$ confluence. Expression of cell surface markers CD29, CD90, CD34, CD45, CD117, HLA-DR and OCT4 was measured in MenSCs and the cells were characterized for their capability to differentiate into adipocytes, osteoblasts and chondroblasts. Culture medium was changed every 3 days. Only passages 6-9 were used for transplantations. MenSCs were stained with $5 \mu \mathrm{L} / \mathrm{mL}$ 1, 1'-dioctadecyl-3,3,3',3'tetramethylindocarbocyanine perchlorate (Dil, SigmaAldrich) at $37^{\circ} \mathrm{C}$ for $20 \mathrm{~min}$ or supplemented with $25 \mu \mathrm{mol} / \mathrm{L}$ 5-bromo-2'-deoxyuridine (BrdU, Sigma) to label MenSCs before transplantation. Cells were harvested with trypsinEDTA (GNM 25200), washed twice with normal saline and resuspended at concentration of $5 \times 10^{5}$ cells $/ \mathrm{mL}$ in normal saline for use. Dil-stained MenSCs were confirmed by fluorescence microscopy (Supplementary Fig. 2).

\section{Group and treatment}

After establishing and confirming the model (Supplementary Fig. $1 C$ and D), the study was started with 131 ICR female mice. The mice were cycle synchronized according to their vaginal smear analysis and divided into two groups before induction of endometrial damage. All the mice were in secretory phase when they were injured and killed for the collection of uterine horns. In MenSCs group $(n=75)$, about $5 \times 10^{5}$ MenSCs in $200 \mu \mathrm{L}$ normal saline were injected via tail vein injection. The control group $(n=56)$ was injected with equal normal saline. Some mice were killed and bilateral uterine horns were resected at Day 3 ( $n=13$ in MenSCs group and $n=12$ in control group), Day 7 ( $n=17$ in MenSCs group and $n=16$ in control group) and Day 14 ( $n=13$ in MenSCs group and $n=11$ in control group) after transplantation for histological staining. Twenty-eight mice in MenSCs group and 14 mice in control group were used for fertility tests at Day 7 after transplantation. During these procedures, some of the mice $(n=7)$ died due to different reasons (infection, bowel obstruction).

\section{Ex vivo fluorescence imaging of Dil-labeled MenSCs in mice with endometrial injury}

After endometrial injury model were set up, the mice were transplanted with normal saline or MenSCs labeled with Dil. After 3-14 days of transplantation, mice in both groups were screened with an in vivo living imaging system (IVIS Spectrum, PerkinElmer) to characterize and visualize Dil-positive cells. The mice were anesthetized and bilateral uteri were resected
A

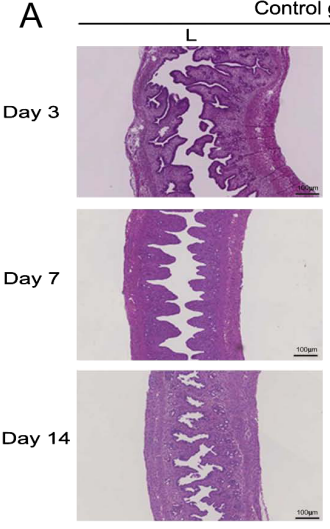

Control group

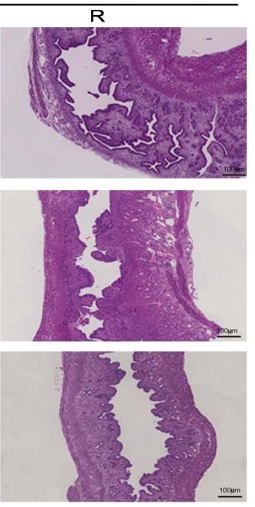

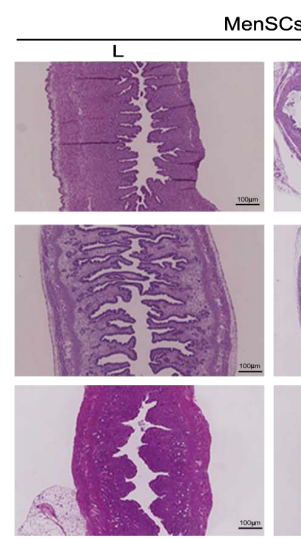

MenSCs group

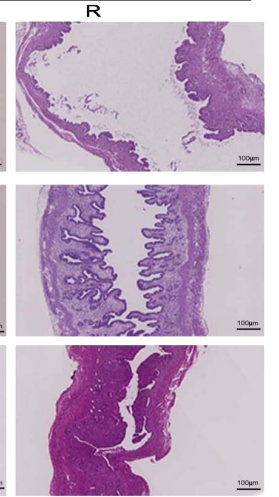

B

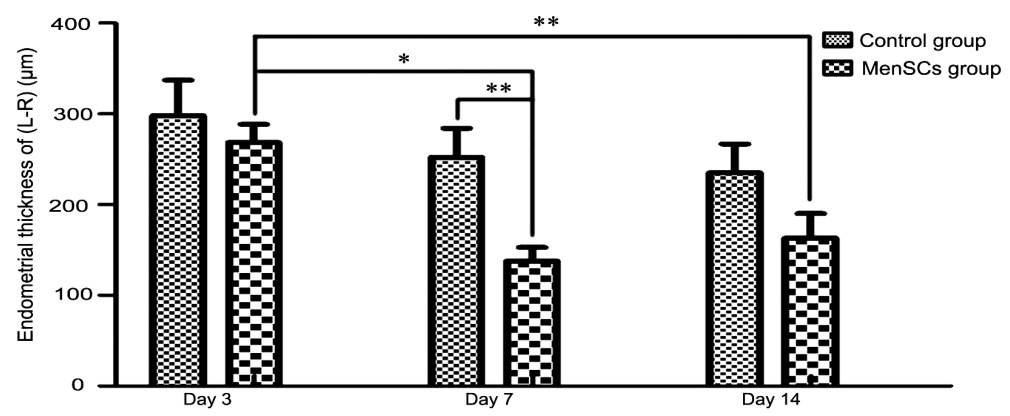

Figure 2 Morphological observations of injured endometrium by HE staining. The right uterine horns of all the female were injured, leaving the left horns untreated as self-controls. Three days after injury, MenSCs or normal saline were transplanted into the injured mice by tail vein injection. Bilateral horns were resected and stained with hematoxylin and eosin (HE) at Day 3 ( $n=13$ in MenSCs group, $n=12$ in control group), Day 7 ( $n=17$ in MenSCs group, $n=16$ in control group) and Day $14(n=13$ in MenSCs group, $n=11$ in control group) after transplantation. The thickness of the endometrium was measured using the Image software. Difference value of the thickness of left horn minus right horn $(\mathrm{L}-\mathrm{R})$ was defined as the extent of restoration of injured endometrium. (A) The representative photos were shown (40x). (B) Bar graph showed that a significantly thicker endometrium in MenSCs group was observed compared with control group at Day 7 after transplantation, with lower difference value. No difference was found between two groups at Day 3 and Day 14 after MenSCs transplantation. In MenSCs groups, injured endometrium at Day 7 and Day 14 was thicker than that at Day 3. However, there was no difference between three time points in control groups. The thickness of endometrium was as mean \pm S.D. One-way ANOVA was applied. ${ }^{*} P<0.05,{ }^{* *} P<0.01$. 
A

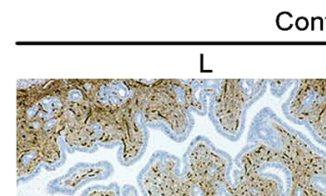

Day 3

$100 \times$

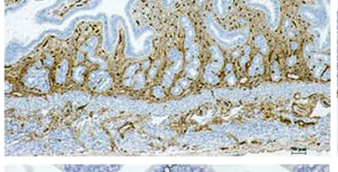

Day 3

$400 \times$
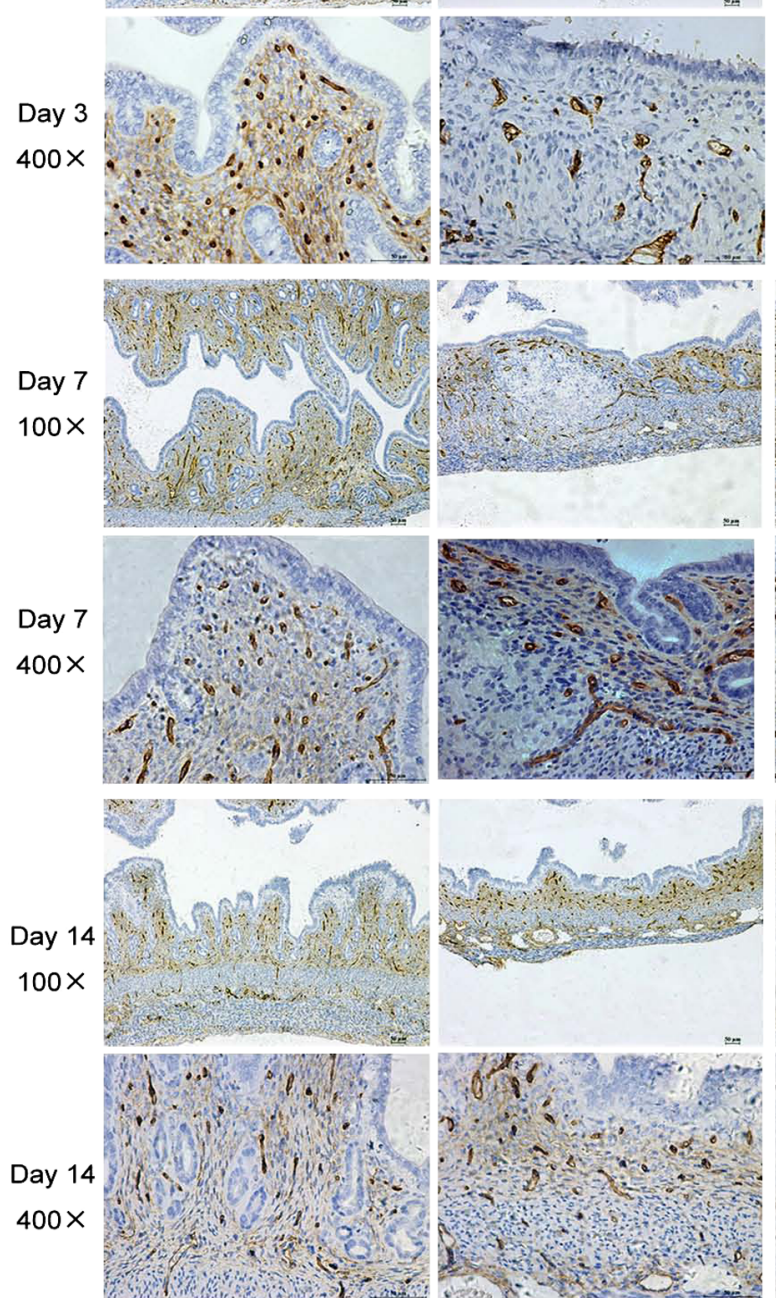

B
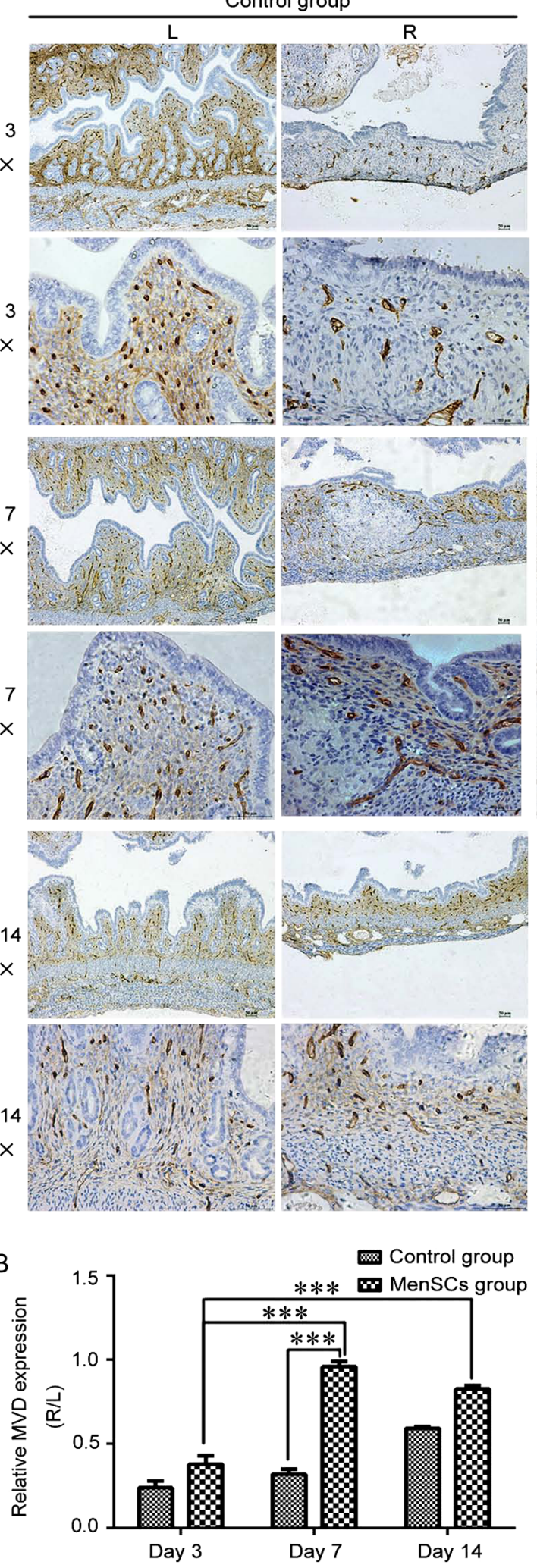
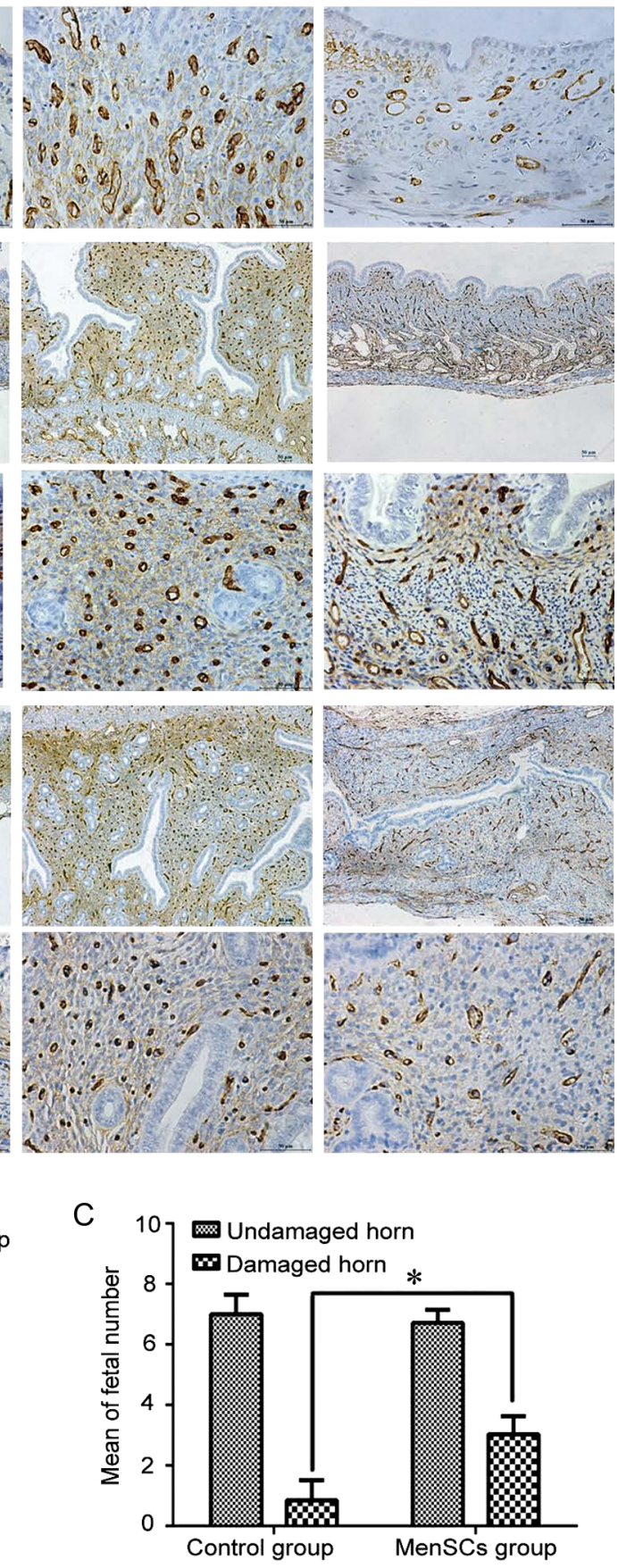

Figure 3 MenSCs could increase MVD and fertility in endometrial injury mice. Collected horns from Day 3, Day 7 and Day 14 after transplantation were immunohistochemically stained with CD34 antibody. (A) The representative photos were shown. (B). Bar graph showed a significant difference between MenSCs group and control group at Day 7 after transplantation $(P=0.000)$. No significant difference was found between groups at Day 3 and Day 14 after transplantation $(P>0.05)$. What is more, relative MVD $(R / L)$ at Day 7 and Day 14 were significantly higher than those at Day 3 in MenSCs groups $(P<0.000)$. The angiogenesis effect was expressed by relative MVD expression $(R / L)$. The MVD was 
to detect the signal at Day 3, Day 7 and Day 14 after transplantation ( $n=3$ per group at each time point) and immediately subjected to fluorescence imaging using an IVIS. Spectrum was coupled with cool charged-coupled device (CCD) camera with excitation wavelength at $549 \mathrm{~nm}$ and emission wavelength at $565 \mathrm{~nm}$. The images and measurements of fluorescence signals were acquired and analyzed with the live imaging software version 4.3 (PerkinElmer). The fluorescence of the left undamaged uterine horn was defined as the background tissue autofluorescence. The resulting images were processed by subtracting the background tissue autofluorescence from the activatable fluorescence with imaging software.

\section{Immunohistochemical staining of CD34 and BrdU}

After fixation in $4 \%$ paraformaldehyde, the uterine horns were embedded in paraffin, and $5 \mu \mathrm{m}$ serial sections were prepared. The sections were deparaffinized in xylene, rehydrated through a series of ethanol washes and rinsed in PBS. Endogenous peroxidase activity was blocked by incubating the sections in $0.3 \% \mathrm{H}_{2} \mathrm{O}_{2}$ for $10 \mathrm{~min}$ at room temperature. The sections were then blocked for $20 \mathrm{~min}$ in PBS with $5 \%$ normal goat serum (YEASEN 36119SE03, China). Then, the slices were examined by incubating with a rabbit monoclonal antibody against cluster of differentiation 34 (CD34 Abcam) at room temperature for $50 \mathrm{~min}$ or BrdU antibody (Sigma-Aldrich) at $4^{\circ} \mathrm{C}$ overnight, followed by horseradish peroxidase-conjugated goat anti-rabbit IgG (Beijing ZSGB biotechnology, China) for $40 \mathrm{~min}$ at room temperature. The sections were then developed with 3,3'-diaminobenzidine (DAB, Maixin Biotech, China), followed by counterstaining with a hematoxylin solution (Beyotime Biotechnology, China) for $3 \mathrm{~min}$. Images were obtained by microscopy. MVD was measured as described by Weidner (1995). Slices were scanned at low power to determine areas of highest vascular density where individual microvessels were counted in five separate random fields at high power $(400 \times)$. The mean vessel count from the five fields was determined and expressed as the MVD (number/ $\mathrm{mm}^{2}$ ). A single countable microvessel was defined as any endothelial cell or group of cells that was clearly separate from other vessels, stroma without the necessity of a vessel lumen or RBC within the lumen. The angiogenesis effect was expressed by MVD expression of right endometrium relative to left endometrium (R/L).

\section{Fertility testing}

To investigate fertility after transplantation of MenSCs or normal saline, the injured female mice were bred with male mice at Day 7 after transplantation for 1 week and then isolated from the males. We observed these female mice every day for the following 2 weeks and found that all the female mice were pregnant without delivery. The pregnancy rate and fetal number of each uterine horn were recorded and assessed.

\section{Culture of human umbilical vein endothelial cells (HUVECs)}

HUVECs were obtained from a commercial source (Science Cell, Carlsbad, CA, USA) and only passages 4-7 were used in experiments. The cells were cultured in basic endothelial culture medium (ECM) (Science Cell) supplemented with $5 \%$ fetal bovine serum (FBS) and endothelial cell growth supplement (Science Cell) at $37^{\circ} \mathrm{C}$ with $5 \% \mathrm{CO}_{2}$. HUVECs were synchronized by incubating for $12 \mathrm{~h}$ in low serum ECM (0.5\% FBS) before cells were used to analyze proliferation, apoptosis, migration and angiogenesis.

\section{Preparation of MenSCs-CM}

MenSCs were seeded at a density of $5 \times 10^{5}$ cells and cultured for $24 \mathrm{~h}$ in the Chang Medium. Then, the medium was replaced with $10 \mathrm{~mL}$ Dulbecco's modified Eagle's medium, and cells were continued to culture for an additional 24 or $48 \mathrm{~h}$. Culture supernatants were collected and centrifuged at $1000 \mathrm{~g}$ for $3 \mathrm{~min}$ to remove cells and cell debris. The clarified supernatant was then concentrated with $3 \mathrm{~K}$ nominal molecular weight limit Filter Device (Merck Millipore) at $4000 \mathrm{~g}$ for $60 \mathrm{~min}$, then was passed through a $0.22 \mu \mathrm{m}$ filter and stored at $-70^{\circ} \mathrm{C}$. The final supernatants from MenSCs (MenSCs-CM) cultured for 24 or 48 $\mathrm{h}(\mathrm{CM}-24 \mathrm{~h}$ or $\mathrm{CM}-48 \mathrm{~h})$ were diluted with ECM before use.

\section{Proliferation assay}

Cell proliferation was determined by Cell Counting Kit 8 assay (CCK8) (GB707, DOJINDO Japan) according to the manufacturer's instructions. Briefly, 1000 HUVECs per well were seeded in a 96-well plate with ECM containing 5\% FBS and incubated for $24 \mathrm{~h}$ in a humidified incubator at $37^{\circ} \mathrm{C}$ for adhesion, followed by synchronization with low serum $(0.5 \%$ FBS) ECM for $12 \mathrm{~h}$. The medium was replaced with $0.5 \%$ FBS $\mathrm{CM}-24 \mathrm{~h}$ or $\mathrm{CM}-48 \mathrm{~h}$ using $0.5 \%$ FBS ECM as control. Then, the absorbance at $450 \mathrm{~nm}$ was measured by a microplate reader for 7 consecutive days. The experiments were performed in triplicate and repeated three times.

\section{Apoptosis assay \\ Apoptosis was assessed with cellular dyes that detect membrane alterations and stain apoptotic cells using apoptosis kit (Alexa Fluor 488 Annexin/Dead Cell Apoptosis Kit, Invitrogen). At 70\% confluence, the apoptosis of HUVECs was induced by $250 \mu \mathrm{mol} / \mathrm{L} \mathrm{H}_{2} \mathrm{O}_{2}$ for $6 \mathrm{~h}$, and then HUVECs were treated by $0.5 \%$ FBS $\mathrm{CM}-24 \mathrm{~h}$ or $\mathrm{CM}-48 \mathrm{~h}$ using $0.5 \%$}

expressed as mean \pm S.D. One-way ANOVA was applied. $* * P<0.01, * * * P<0.001$. (C) MenSCs or normal saline were injected into mice via the tail vein at Day 3 after the right uterine horn was injured by electrocoagulation. At Day 7 after transplantation, the injured female mice were bred with male mice for 1 week and then isolated from the males. Three weeks after mating, all the female mice were pregnant without delivery. Fetal numbers in damaged and undamaged horns in both MenSCs group $(n=28)$ and control group ( $n=14)$ were counted and expressed as mean \pm S.D. and displayed in the bar graph. Unpaired Student's $t$-test was applied. ${ }^{*} P<0.05$. 
A

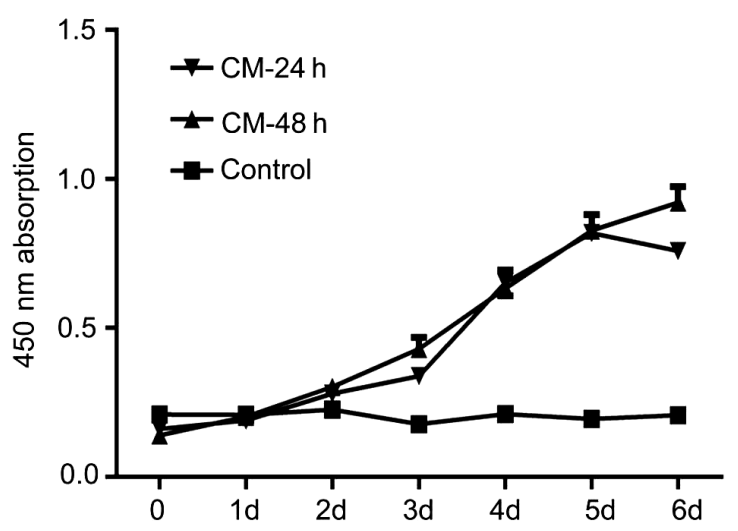

B

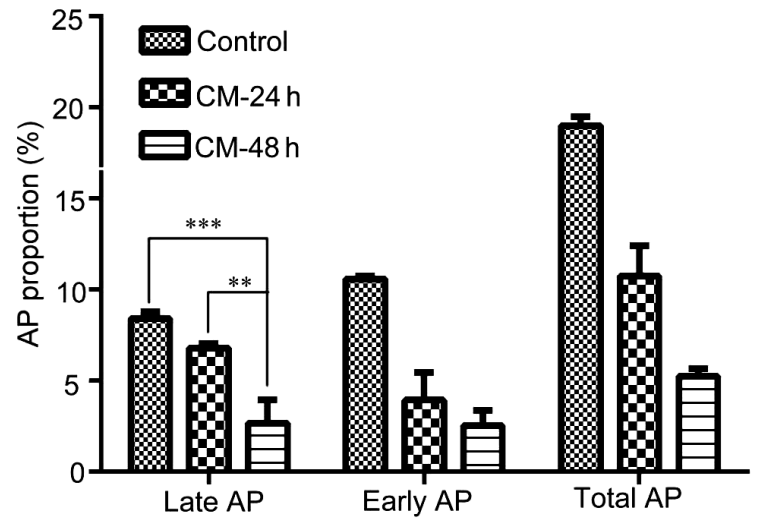

C
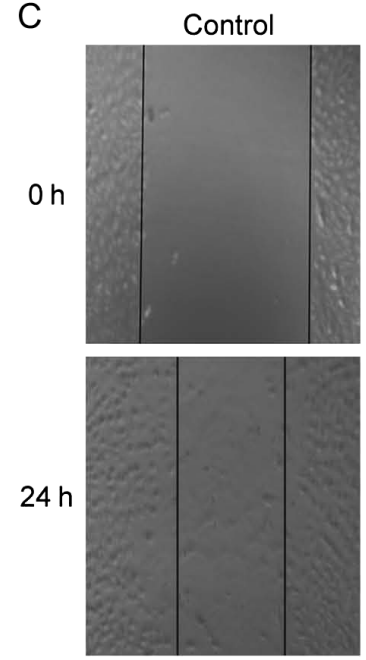

E

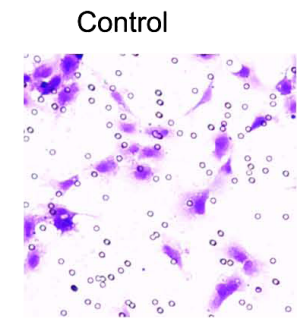

G

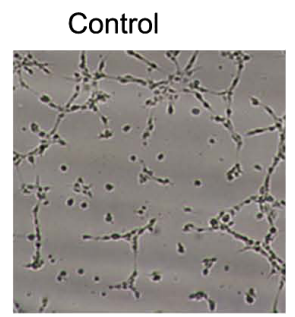

CM-24h
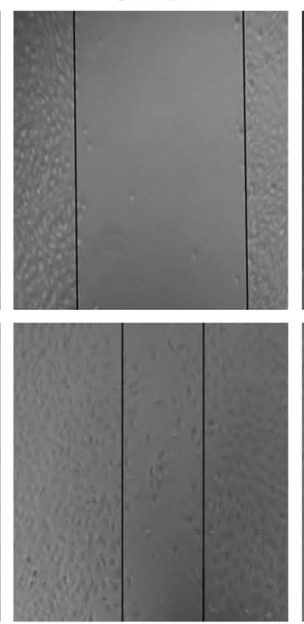

CM-24 h

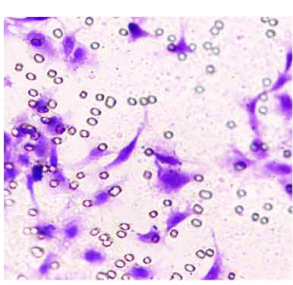

$\mathrm{CM}-24 \mathrm{~h}$

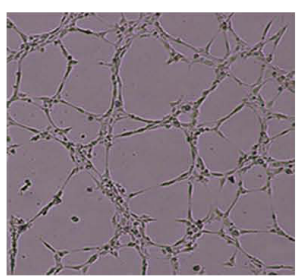

CM-48 h

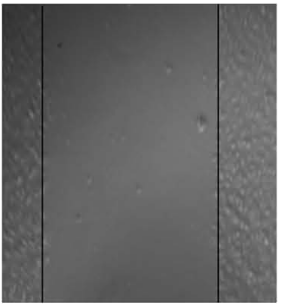

CM-48 h

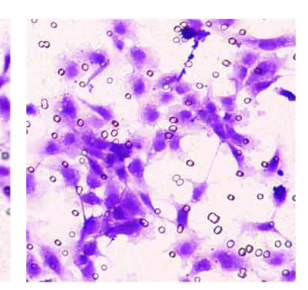

CM-48 h

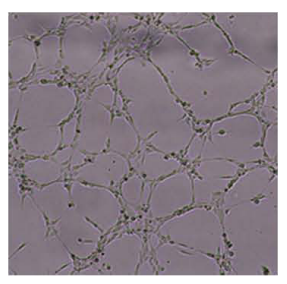

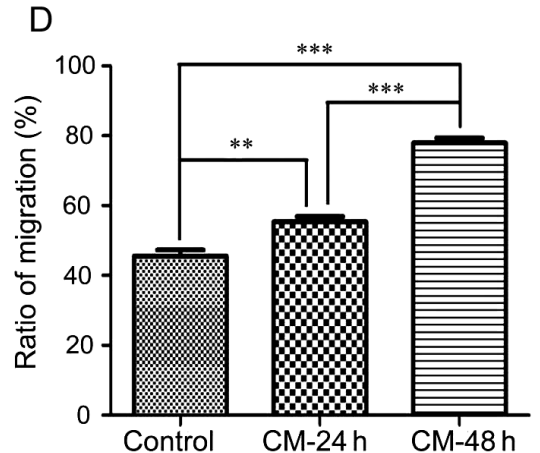

F

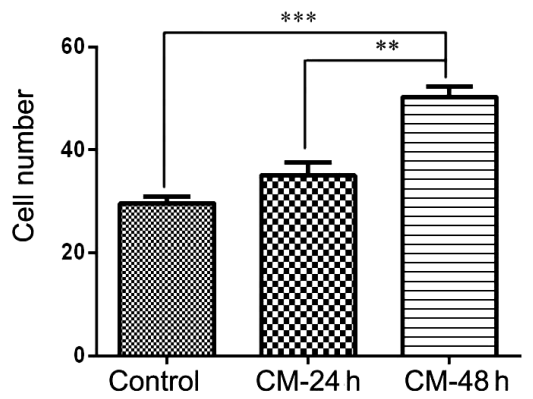

$\mathrm{H}$

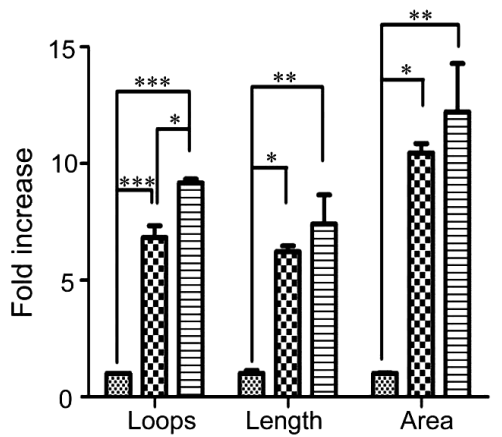

Figure 4 MenSCs-CM inhibits $\mathrm{H}_{2} \mathrm{O}_{2}$-induced apoptosis and promotes proliferation, migration and angiogenesis of HUVECs. (A) CM-24h and CM-48 h could promote the proliferation of HUVECs. CCK8 assay was applied to assess the proliferative ability of HUVECs treated with CM-24 $\mathrm{h}$ or CM-48 h using HUVECs basic culture medium ECM as control. The experiments were performed in triplicate and repeated three times. (B) Apoptosis of HUVECs was inhibited after treatment with CM-24h or CM- $48 \mathrm{~h}$ for $24 \mathrm{~h}$. The apoptosis of HUVECs was induced by $250 \mu \mathrm{mol} / \mathrm{mL}$ $\mathrm{H}_{2} \mathrm{O}_{2}$ for $6 \mathrm{~h}$ and then HUVECs were treated by $\mathrm{CM}-24 \mathrm{~h}$ or $\mathrm{CM}-48 \mathrm{~h}$ using ECM as control. AP denotes apoptosis. All samples were assayed 

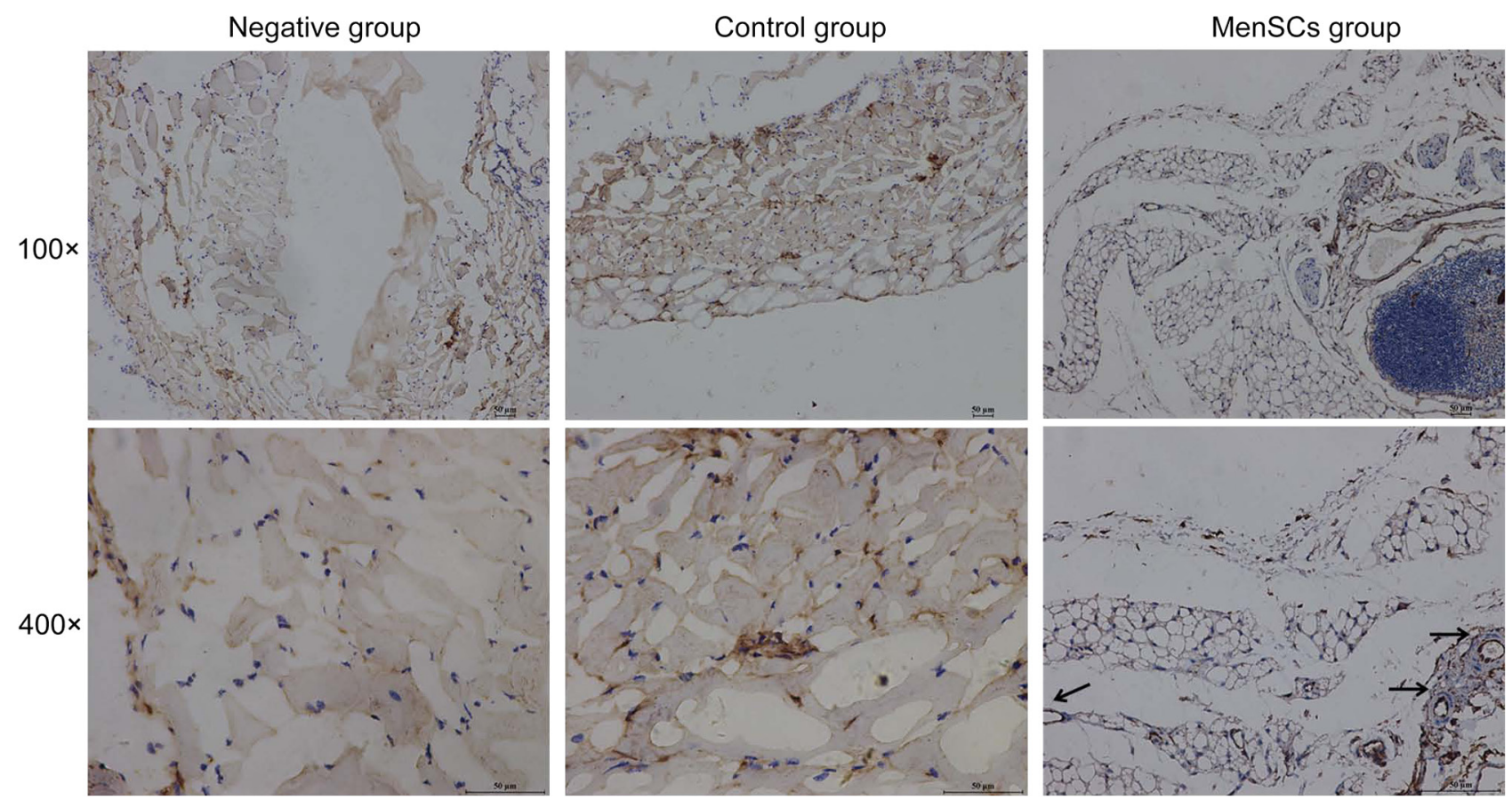

Figure 5 MenSCs-CM promotes endothelial cell migration and tube formation in vivo. Six-week-old C57/BL/6 mice were subcutaneously injected with $500 \mu \mathrm{L}$ Matrigel containing CM-48 h (concentration 50x) using PBS as negative control and ECM as control into the ventral area ( $n=5$ per group). After 6 days, the skin of mice was pulled back to expose the intact Matrigel plugs. The removed Matrigel plugs were fixed with an optimal cutting temperature (OCT) compound, and 6- $\mu$ m-thick fresh sections were made for IHC with CD34 antibody to identify the migration of the endothelial cells and infiltration of new microvessels. More vessels and migrated endothelial cells were found in MenSCs group compared with negative group and control group. And no migrated endothelial cells in negative group and only few migrated endothelial cells in control group were observed.

FBS ECM as control for $24 \mathrm{~h}$. After treatment, HUVECs were double stained with propidium iodide (PI) and fluorescein isothiocyanate-conjugated annexin $\mathrm{V}$, and then analyzed by flow cytometry. Pl-/annexin $\mathrm{V}+$ indicated early apoptotic cells, $\mathrm{PI}+$ /annexin $\mathrm{V}+$ indicated late apoptotic cells and $\mathrm{PI}+$ /annexin $\mathrm{V}$ - indicated dead cells. Total apoptotic cells were the sum of early and late apoptotic cells. All samples were assayed with three copies and repeated three times.

\section{Migration assay}

The migration of HUVECs was assessed by wound healing assay and transwell migration assay. For wound healing assay, at $100 \%$ confluence, the monolayer was scratched with a sterile $200 \mu \mathrm{L}$ pipette tip, washed and then incubated with $0.5 \%$ FBS $\mathrm{CM}-24 \mathrm{~h}$ or $\mathrm{CM}-48 \mathrm{~h}$ using low serum ECM as control for $24 \mathrm{~h}$. Images were obtained with a digital camera at 0 and $24 \mathrm{~h}$, and analyzed manually with Image software. The ratio of migration was calculated by the distance of migration/ the distance between monolayers at $0 \mathrm{~h}$. The results were obtained from three separate experiments that were performed in triplicate.

For transwell migration assay, serum-starved HUVECs were trypsinized and cells were resuspended with serum-free CM-24 h, CM-48 h or ECM (control) and added into the upper well of a transwell filter apparatus. The filter is suspended in a well of a 24-well plate and the lower reservoir is filled with the DMEM with $10 \%$ FBS. The cells are incubated under normal conditions for $24 \mathrm{~h}$. Nonmigrated cells from top surface were rubbed off and cells that had migrated through the membrane were fixed with $75 \%$ alcohol for $10 \mathrm{~min}$ and stained with crystal violet. The stained cells were observed

with three copies. One-way ANOVA was applied. ${ }^{* *} P<0.01,{ }^{* * *} P<0.001$. The experiments were performed in triplicate and repeated three times. (C) MenSCs-CM increased HUVECs migration by wound healing assay. HUVECs treated with CM-24h, CM-48 h or ECM (control) were subjected to wound healing assay for $24 \mathrm{~h}$. Representative images are shown $(40 \times)$. All the experiments were performed in triplicate and repeated three times. (D) Quantitative analysis of the ratio of migration. The values are expressed as the distance of migration/the distance between monolayers at $0 \mathrm{~h}$. The data were obtained from three independent experiments. One-way ANOVA was applied. ${ }^{* *} P<0.01$, ${ }^{* * *} P<0.001$. (E) MenSCs-CM increased HUVECs migration by transwell migration assay. CM-24 $\mathrm{h}$ and $\mathrm{CM}-48 \mathrm{~h}$ promoted HUVECs migration compared with control group. All the experiments were performed in triplicate and repeated three times. (F) Quantitative analysis of the number of migrated HUVECs. The data were obtained from three independent experiments. One-way ANOVA was applied. ${ }^{* *} P<0.01,{ }^{* * *} P<0.001$. (G) MenSCs-CM improved the tube formation ability of HUVECs. After treatment with CM-24h, CM-48 h or ECM (control) for $4 \mathrm{~h}$, formed vessel-like structures of HUVECs were photographed (40x). All experiments were assayed with five copies and repeated three times. $(\mathrm{H}) \mathrm{Quantitative}$ analysis of loop numbers, tube length and area. Values were calculated by ImageJ software and the value in control group was defined as 1 . The data were obtained from three independent experiments. One-way ANOVA was applied. ${ }^{*} P<0.05,{ }^{* *} P<0.01$, and ${ }^{* * *} P<0.001$. 

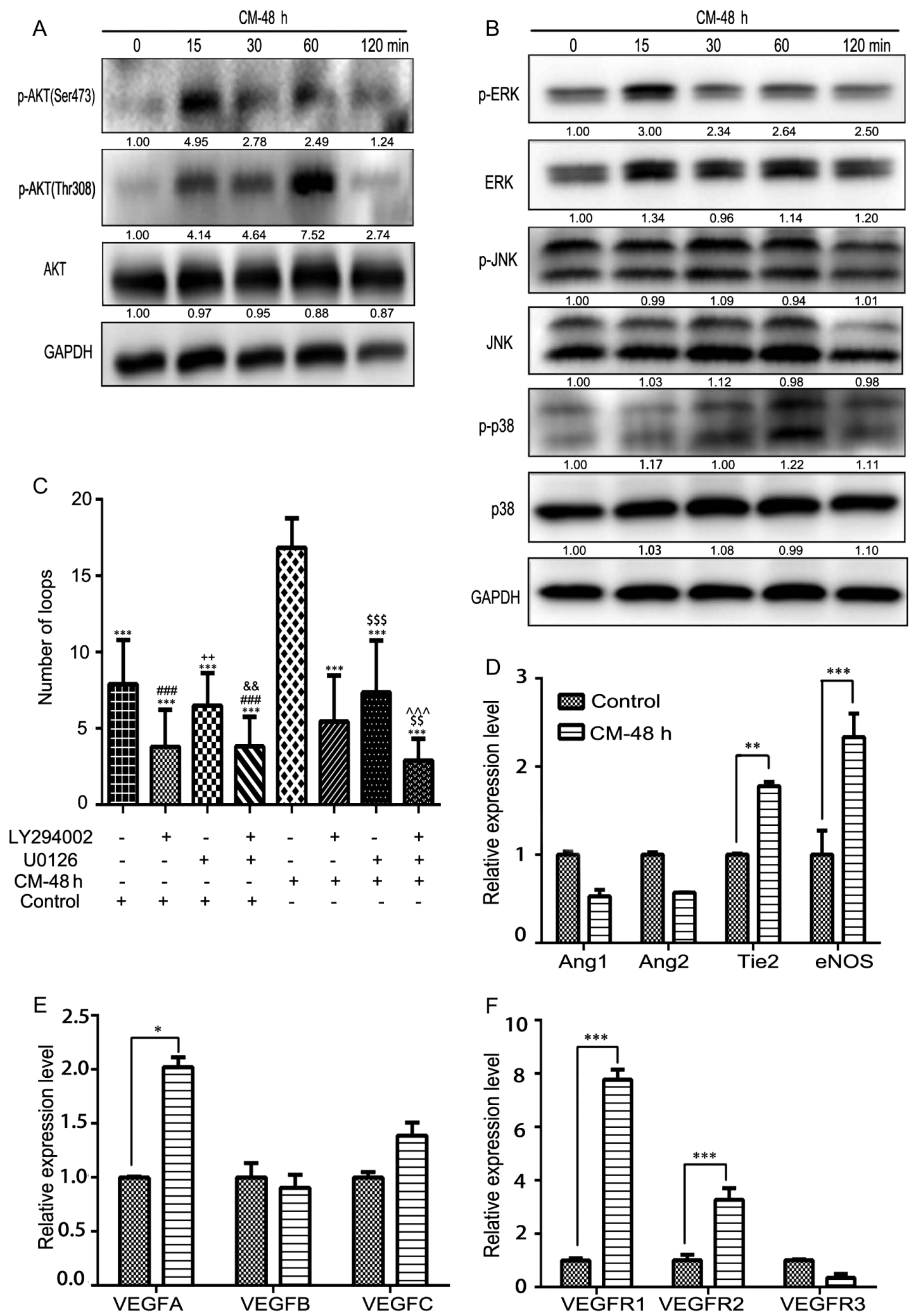

Figure 6 MenSCs-CM activates HUVECs AKT and ERK signaling pathways. (A and B) The protein levels of p-AKT, AKT, p-ERK, ERK, p-JNK, JNK, p-p38 and p38 in HUVECs were detected by Western blotting after cocultured with CM-48 h for indicated period $(n=3)$. The densitometric analysis of Western blotting bands was shown below of each band. (C). Bar graph shows quantitative analysis of tube formation in each group. HUVECs were pretreated with LY294002 $(20 \mu \mathrm{mol} / \mathrm{L})$ and U0126 $(10 \mu \mathrm{mol} / \mathrm{L})$ for $1 \mathrm{~h}$ before the cells were used for Matrigel angiogenesis analysis. All experiments were assayed with three copies and repeated three times. One-way ANOVA was applied. ${ }^{\sharp \sharp} P<0.001$ vs control 
under a microscope, and at least five fields of cells were assayed for each group. All experiments were assayed in triplicate and repeated three times.

\section{Matrigel tube formation assay}

Matrigel tube formation assays were performed to assess in vitro angiogenesis using $\mu$-Slide Angiogenesis plate (ibidi, Martinsried, Germany) and Matrigel (BD Bioscience, San Jose, CA, USA). Briefly, HUVECs ( $10^{4}$ cells/well) were cultured in the $\mu$-Slide Angiogenesis plate coated with Matrigel and incubated with $0.5 \%$ FBS CM-24h or $0.5 \%$ FBS CM-48h or low serum ECM with or without PI3K inhibitor (LY294002, Cell Signaling Technology) or ERK inhibitor (U0126, Cell Signaling Technology) in triplicate. Tube-like structures in each well were captured under a light microscope. The number of loops (the formed tubes), the length and the area of the formed tubes were scanned and quantitated with ImageJ software. All experiments were assayed with five copies and repeated three times.

\section{In vivo mice Matrigel plug assay}

Matrigel plug assay was performed as described by Malinda (2003). $0.5 \mathrm{~mL}$ Matrigel containing $50 \mu \mathrm{L} \mathrm{CM}$ (concentration $50 \times$, MenSCs group) or ECM (control group) or PBS (negative group) were injected subcutaneously into the ventral area of C57/BL/6 mice ( $n=5$ per group). After 7 days, the mice were killed and intact Matrigel plugs from three groups of mice were removed. The removed Matrigel plugs were photographed and were fixed with an optimal cutting temperature (OCT) compound, and $6 \mu \mathrm{m}$-thick fresh sections were made for IHC staining to identify the migration of the endothelial cells and infiltration of new microvessels. Slides were washed twice with PBS and incubated with $5 \%$ bovine serum albumin (BSA) for $30 \mathrm{~min}$ at room temperature. Sections were incubated overnight at $4^{\circ} \mathrm{C}$ with CD34 antibody (Abcam). At least three random fields of the area underlying the skin of each mouse were counted.

\section{Western blotting}

After treatment with CM-48h, HUVECs were lysed in RIPA buffer for $30 \mathrm{~min}$. The lysate was centrifuged at $12,000 \mathrm{~g}$ for $20 \mathrm{~min}$ at $4^{\circ} \mathrm{C}$. The supernatant was collected and the protein concentration was determined using a BSA protein assay. For the detection of phosphorylated and total AKT, p38, ERK and JNK, proteins from each group were subjected to $12 \%$ sodium dodecyl sulfate polyacrylamide gel electrophoresis and then transferred to a polyvinylidenefluoride membrane. The membranes were blocked with 5\% dry milk in Tris-buffered saline with $0.1 \%$ TWEEN (TBST) and then incubated overnight at $4{ }^{\circ} \mathrm{C}$ with primary antibodies. The primary antibodies were purchased from Cell Signaling Technology against p-Akt (1:1000; Ser473), p-Akt (1:1000; Thr308), Akt (1:1000), p-ERK (1:1000; Thr202/Tyr204), ERK (1:1000), ERK (1:1000), p-p38 (1:500), p38 (1:1000), p-JNK (1:500) or JNK (1:1000). The membrane was washed with TBST and then incubated with anti-rabbit secondary antibodies (1:2000). Immunoreactivity was detected using enhanced chemiluminescence. Equal amounts of protein were verified by reprobing the membrane with an anti-glyceraldehyde-3-phosphate dehydrogenase (GAPDH) antibody.

\section{Quantitative reverse transcription polymerase chain reaction ( $q R T-P C R)$}

Total RNA was extracted from cells using TRIzol reagent (Invitrogen). cDNA synthesis was conducted in a $25 \mu \mathrm{L}$ reaction mixture using Reverse Transcriptase M-MLV and random primers (Promega) according to the manufacturer's instructions. cDNA was analyzed by qRT-PCR using Bestar Real time PCR Master Mix (DBI Bioscience, Germany) following the manufacturer's instructions and primers for angiogenesisrelated genes (Supplementary Table 1). The amplification program consisted of an initial denaturation step at $95^{\circ} \mathrm{C}$ for $30 \mathrm{~s}$ followed by 40 cycles of denaturation at $95^{\circ} \mathrm{C}$ for $15 \mathrm{~s}$, annealing at $60^{\circ} \mathrm{C}$ for $30 \mathrm{~s}$ and extension at $72^{\circ} \mathrm{C}$ for $30 \mathrm{~s}$. The total quantity of fluorescent product was measured at the end of every cycle with a single acquisition. GAPDH was used as the internal control. The relative expression level of target gene $\left(2^{-\Delta \Delta \mathrm{Ct}}\right)$ was normalized to the endogenous GAPDH reference $(\Delta \mathrm{Ct})$ and related to the amount of target gene in control group, which was defined as the calibrator at 1 .

\section{Statistical analysis}

The data are presented as the mean \pm standard deviation (S.D.) of at least three separate experiments. Statistical comparisons between two groups were performed by student's t-test. Multiple groups were analyzed by one-way ANOVA followed by appropriate post hoc test to determine statistical significance. The data in Table 1 were analyzed by chi-square test. All in vitro experiments were repeated at least in triplicate and analyzed. A $P$ value of less than 0.05 was considered statistically significant. All statistical analyses were performed using SPSS 16.0 for Windows (SPSS 16.0) with two-tailed tests.

\section{Results \\ MenSCs engraft in the mouse model of uterine damage}

To investigate the influence of endometrial injury on the migration of MenSCs to the endometrium, the right horns of female mice were injured by

medium group, ${ }^{* * *} P<0.001$ vs $C M-48$ h group, ${ }^{++} P<0.01$ vs control medium in combination with LY294002, $\$ \$ P<0.01, \$ \$ \$ P<0.001$ vs CM-48 h medium in combination with LY294002, \&\& $P<0.01$ vs control medium in combination with $\cup 0126,{ }^{\wedge \wedge} P<0.001$ vs $C M-48 \mathrm{~h}$ medium in combination with U0126. (D-F) HUVECs were incubated in low serum ECM (0.5\% FBS) for $12 \mathrm{~h}$ and then were treated by CM-48 $\mathrm{h}$ for $24 \mathrm{~h}$. RNA was extracted from the treated cells for qRT-PCR analysis. Quantitative analysis of angiogenesis-related factors was shown. Tie2, eNOS, VEGFA, VEGFR1 and VEGFR2 were increased significantly in all CM-48 hroups in comparison with control group. All experiments were assayed with three copies and repeated three times. Unpaired Student's $t$-test was applied. ${ }^{*} P<0.05,{ }^{* *} P<0.01$, and ${ }^{* * *} P<0.001$. 
electrocoagulation as described above, leaving the left horns untreated as self-controls. Three days after injury, the mice were intravenously injected with Dil-labeled MenSCs or normal saline. At Day 3, Day 7 and Day 14 after injection, bilateral horns in both groups were collected, and fluorescent signals were detected through IVIS to determine MenSCs recruitment ( $n=3$ per group at different time point). As expected, fluorescence was only detected in injured horns of the MenSCs groups (Fig. 1), while no fluorescent signal was seen in control groups and undamaged horns in MenSCs group. We further microscopically confirmed that MenSCs could home and integrate into the damaged uterine tissue by immunohistochemistrical staining with BrdU antibody on uterine sections at Day 3, Day 7 and Day 14 after transplantation ( $n=3$ per group at different time point, Supplementary Fig. 3).

\section{MenSCs treatment is beneficial to restore injured endometrium by increasing endometrial thickness and MVD}

To explore the effect of MenSCs transplantation on the injured endometrium, we assessed the difference value of endometrial thickness (L-R) between two horns and MVD of the right relative to the left (R/L). MVD has been widely applied in the assessment of angiogenesis (Chen et al. 2013). At Day 3 after transplantation, there was no difference in the endometrial thickness $(\mathrm{L}-\mathrm{R})$ and the relative MVD (R/L) between two groups $(298.26 \pm 135.15 \mu \mathrm{m}$ vs $268.46 \pm 72.19 \mu \mathrm{m}, \quad P=0.414$ for thickness, $0.24 \pm 0.04$ vs $0.38 \pm 0.05, P=0.058$ for MVD, Figs 2, 3A and B). However, after 7 days after transplantation, a significant difference in the endometrial thickness (L-R) and MVD (R/L) was observed between two groups $(252.12 \pm 78.58 \mu \mathrm{m}$ vs $137.97 \pm 61.56 \mu \mathrm{m}$, $P=0.010$ for thickness, $0.32 \pm 0.03$ vs $0.96 \pm 0.03$, $P=0.000$ for MVD, Figs 2, 3A and B). At Day 14 after transplantation, endometrial thickness (L-R) in MenSCs group was lower than those in control group, although the difference was not significant $(235.22 \pm 89.57 \mu \mathrm{m}$ vs $163.24 \pm 97.06 \mu \mathrm{m}, P=0.082$, Fig. $2 \mathrm{~B}$ ). In terms of $M V D$, there was no statistical significance between two groups at Day 14 after transplantation. Histological evaluation of the endometrium at Day 7 and Day 14 after MenSCs transplantation revealed an intact structure of the endometrial layer characterized by an increased endometrial thickness and capillaries (Fig. 2A). It was worth mentioning that the endometrial thickness (L-R) in MenSCs group presented a decreasing trend $(P<0.05$, Fig. 2B) during 14 days, whereas no difference was found in control groups. What is more, MVD $(\mathrm{R} / \mathrm{L})$ at Day 7 and Day 14 was significantly higher than that at Day 3 in MenSCs groups $(P<0.000)$. These results indicated that MenSCs indeed promote the restoration of the damaged endometrium, possibly through the proangiogenesis effect of MenSCs.

\section{MenSCs treatment improves fertility of mice with the injured endometrium}

To investigate the effect of MenSCs on fertility in endometrial injury mice, the right uterine horns of the female mice were injured, and all the injured mice were transplanted with MenSCs $(n=28)$ or normal saline $(n=14)$. The operated female mice were bred with male mice for 1 week from 7 days after transplantation, and then isolated from the males. We observed these female mice every day for additional 2 weeks and found that all the female mice were pregnant without delivery. While conception occurred in all the undamaged horns in both groups, 15 of 28 damaged horns in MenSCs group and only 2 of 14 damaged horns in control group conceived $(P=0.014$, $\chi^{2}$, Table 1). The fetal number of the undamaged horns in MenSCs group were similar to that in control group $(P=0.830)$. However, a significant difference in the embryo number in damaged horns were found between MenSCs group and control group (3.1 \pm 0.6 vs $0.9 \pm 0.7, P=0.030$, Fig. $3 \mathrm{C}$ ).

\section{MenSCs-CM suppresses $\mathrm{H}_{2} \mathrm{O}_{2}$-induced apoptosis and promotes proliferation, migration and angiogenesis}

As paracrine action has been considered as the predominant mechanism for the role of MSCs in tissue repair (Camussi et al. 2010), we collected MenSCs-CM cultured for 24 or $48 \mathrm{~h}(\mathrm{CM}-24 \mathrm{~h}$ or $\mathrm{CM}-48 \mathrm{~h})$ and treated HUVECs with MenSCs-CM to verify the above results that MenSCs could increase MVD of injured endometrium. To determine the effect of MenSCs-CM on HUVECs proliferation, we evaluated the viability of HUVECs treated with CM-24 $\mathrm{h}$ and $\mathrm{CM}-48 \mathrm{~h}$ respectively by proliferation and apoptosis assays using HUVECs basic culture medium ECM as control. The results of CCK8 assays showed that both CM-24h and CM-48 h could promote HUVECs growth compared with control group (Fig. 4A). Further, MenSCs-CM could block $\mathrm{H}_{2} \mathrm{O}_{2}$ induced late apoptosis of HUVECs, and the inhibiting effect of CM-48 $\mathrm{h}$ was even stronger than that of $\mathrm{CM}-24 \mathrm{~h}$ $(P=0.003$, Fig. 4B).

Next, we detected whether HUVECs migratory ability was affected by MenSCs-CM via wound healing assays and transwell migration assays. HUVECs were incubated with $\mathrm{CM}-24 \mathrm{~h}$ or $\mathrm{CM}-48 \mathrm{~h}$, using $\mathrm{ECM}$ as control. The results exhibited that both $\mathrm{CM}-24 \mathrm{~h}$ and $\mathrm{CM}-48 \mathrm{~h}$ could enhance the wound closure rate, and the migration of HUVECs treated with CM-48h was faster than that of cells treated with $\mathrm{CM}-24 \mathrm{~h}$ and control $(P<0.001$, Fig. $4 \mathrm{C}$ and $\mathrm{D})$. To exclude the possibility that faster wound closure rate in $\mathrm{CM}-24 \mathrm{~h}$ and $\mathrm{CM}-48 \mathrm{~h}$ groups might be the result of proliferation of HUVECs by MenSCs-CM, we performed transwell migration assays and found that MenSCs-CM indeed promoted HUVECs migration (Fig. 4E and F). 
Furthermore, the potential proangiogenesis effect of MenSCs-CM was evaluated in Matrigel tube formation assays. As presented in Fig. 4G, neocapillary networks with well-formed lumens were observed within $4 \mathrm{~h}$ after seeding the cells on Matrigel, and the formation of vascular-like networks was remarkably enhanced by MenSCs-CM treatment. Quantitative analysis of loop numbers, tube length and area showed that MenSCs-CM improved HUVECs angiogenesis $(P<0.05$, Fig. $4 \mathrm{H})$.

The biological activity of MenSCs on endothelial cells migration and tube formation was also confirmed in Matrigel plug assay in mice. More vessels and migrated endothelial cells were observed in MenSCs group compared with negative group and ECM group. Also, no migrated endothelial cells in negative group and only few migrated endothelial cells in control group were observed (Fig. 5).

\section{MenSCs-CM activates AKT and ERK pathways of endothelial cells and increases their downstream target genes expression}

AKT and MAPK signal pathways play a vital role in angiogenesis (Huang et al. 2015, Zeng et al. 2015, Lv et al. 2016). In this study, we also found that phosphorylated level of AKT and ERK in HUVECs increased notably after treatment with CM-48h (Fig. 6A and B). However, the phosphorylated level of JNK and p38 was not significantly changed after treatment with CM-48 h (Fig. 6B). Since MenSCs-CM could activate AKT and ERK, we next examined whether the angiogenesis induced by MenSCs-CM could be reversed by LY294002 (AKT inhibitor) and/or U0126 (ERK inhibitor). HUVECs were pretreated with LY294002 $(20 \mu \mathrm{mol} / \mathrm{L})$ and U0126 $(10 \mu \mathrm{mol} / \mathrm{L})$ for $1 \mathrm{~h}$ before the cells were used for Matrigel angiogenesis analysis (Supplementary Fig. 4). The results showed that most blood vessels were found in $\mathrm{CM}-48 \mathrm{~h}$ group $(P<0.001$, Fig. 6C and Supplementary Fig. 5). Moreover, angiogenesis by $\mathrm{CM}-48 \mathrm{~h}$ could be blocked by the treatment of LY294002 and U0126 independently or synergistically and the inhibitory effect of LY294002 was stronger than U0126 $(P<0.001$, Fig. $6 \mathrm{C}$ and Supplementary Fig. 5).

Furthermore, qRT-PCR was applied to determine the transcriptional levels of downstream targets of AKT and ERK, including VEGF family genes, Ang family genes and eNOS, all of which were also related to angiogenesis. The results showed that the expression of Tie2, eNOS, VEGFA, VEGFR1 and VEGFR2 was increased significantly in all MenSCs-CM group in comparison with control group $(P<0.05$, Fig. 6D$F)$. These data suggested that MenSCs-CM could induce the overexpression of angiogenesis-related factors, consistent with the findings that MenSCs-CM promoted growth, migration and angiogenesis of endothelial cells.

\section{Discussion}

Recently, a case report showed that intrauterine administration of autologous BMSCs could repair the injured endometrium and result in subsequent pregnancy in a woman with severe IUAs, which was not responding to TCRA followed by the placement of IUD and cyclical hormonal therapy (Nagori et al. 2011). Another study presented that autologous stem cell transplantation led to endometrial regeneration reflected by restoration of menstruation in five out of six patients with refractory Asherman's syndrome (Singh et al. 2014). Lately, several animal studies have demonstrated that MSCs could repair the injured endometrium, but the repair mechanisms of MSCs were not investigated (Alawadhi et al. 2014, Jing et al. 2014, Kilic et al. 2014). Based on these studies, here we established a mouse endometrial injury model by electrocoagulation to evaluate the therapeutic effects of stem cells, which was more easily performed than the mechanical methods described by Alawadhi and coworkers (2014), (Hu et al. 2014). More important, we explored repair mechanisms of stem cells in vitro and in vivo.

With the potential of restoring both tissue structures and functions, stem cells have emerged as a vital element in regenerative medicine. Of all types of MSCs, MenSCs is a noninvasively obtained and ethically appropriate autologous stem cell source. Besides, MenSCs showed higher expression of genes involved in vasculogenesis, angiogenesis, proliferation and migration, all contributing to endometrial function in comparison with BMSCs (Gaafar et al. 2014). Furthermore, numerous preclinical studies have revealed that administration of MenSCs to injured tissues in situ or via intravenous injection induced structural restoration as well as functional improvements in heart failure (Bockeria et al. 2013, Jiang et al. 2013) and critical limb ischemia (Murphy et al. 2008). The similar outcomes were observed in several clinical studies (Zhong et al. 2009, Ichim et al. $2010 a, b)$. However, there was no report about MenSCs transplantation for the treatment of IUAs, which was an intractable gynecological disease in assisted reproductive technology. Our study was the first to document positive effects of MenSCs on the restoration of injured endometrium in mouse endometrial injury models, and we further explored the possible therapeutic mechanisms behind these beneficial effects.

As endometrial thickness significantly affects clinical pregnancy and live birth rate in embryo transfer cycle (Kovacs et al. 2003, Levens et al. 2006, Fang et al. 2016), we measured the endometrial thickness in our animal study. In our mouse endometrial injury model, we found that the MenSCs-treated mice had remarkably increased endometrial thickness 7 days after MenSCs injection, whereas the endometrium of control group recovered slowly. However, there was no significant difference in the endometrial thickness between two groups 14 days 
after grafting, which indicated that MenSCs indeed have the potential to accelerate the restoration and regeneration of the damaged endometrium in agreement with Jing's study (Jing et al. 2014). Our study also showed higher pregnancy rate and more fetal numbers in injured horn of MenSCs-treated mice than that of control group, which was similar to the BMSCs transplantation trial reported by Alawadhi and coworkers 2 years ago (Alawadhi et al. 2014). Above all, our data suggested that treatment with MenSCs could promote endometrial restoration and improve mice fertility after uterine injury.

Therapeutic angiogenesis, defined as the use of biological agents or bioactive materials to stimulate the growth of new blood vessels, has been developed as a way to treat the injured tissue (Thompson et al. 2000, Dragneva et al. 2013). MVD, an objective indicator of newly formed vessels, has been widely applied in the assessment of angiogenesis. CD34 is a specific marker for endothelial cells and has been regarded as the most sensitive marker to detect microvessels (Chen et al. 2013). In our study, we investigated whether MenSCs could promote angiogenesis in vivo and in vitro to treat endometrial injury. Consistent with the study of Chen and coworkers (2013), we found that newly formed blood vessels in MenSCs group was significantly higher than control group in mice endometrial injury model, indicating that MenSCs could promote revascularization and angiogenesis in the injured endometrium during endometrial repair. Angiogenesis was a process that involved endothelial cells proliferation, migration and the formation of new vessels from pre-existing capillaries (Thompson et al. 2000, Dragneva et al. 2013). In vivo Matrigel plug assay found more migrated endothelial cells and formed vessels in MenSCs group than negative group and control group, and further supported the findings in the mice endometrial injury model. In vitro, we found that MenSCs-CM could promote HUVECs angiogenesis, an observation in part explained by influence of MenSCs-CM on cells proliferation, apoptosis and cells migration. Also, CM-48 h was more effective to enhance the biological functions of HUVECs than CM-24h, indicating that MenSCs might continuously secrete angiogenesis-enhancing factors into the culture medium to affect HUVECs migration and neovascularization. Several studies had found that stem cells could paracrine a panel of cytokines and growth factors, contributing to repair of injured tissues (Kinnaird et al. 2004a, Boomsma \& Geenen 2012, Rahbarghazi et al. 2013). AKT and MAPK signal pathway had been proved to play a vital role in angiogenesis (Huang et al. 2015, Zeng et al. 2015, Lv et al. 2016). Interestingly, we found that AKT and ERK could be activated by MenSCs-CM and the transcriptional expression level of eNOS, VEGFA, VEGFR1, VEGFR2 and TIE2 in HUVECs, which are the downstream targets of AKT and ERK pathway, increased after MenSCs-CM treatment. These results could partially clarify the mechanisms of improved angiogenesis mediated by MenSCs-CM. In sum,
MenSCs could promote tube formation of endothelial cells in vitro and angiogenesis in a mouse endometrial injury model and in vivo Matrigel plug assay. Therapy with autologous MenSCs held tremendous potential to accelerate reparative processes of injured endometrium. However, certain problems need to be resolved before it became routine clinical practice. Some details such as dose of transplanted cells and route of administration need to be optimized. Moreover, numerous other mechanisms may also be involved in the restoration of injured endometrium by MenSCs. Therefore, further studies are required to elucidate the effects of MenSCs during tissue restoration.

\section{Conclusions}

Our study demonstrated that MenSCs could help repair the injured endometrium and improve fertility in a mouse endometrial injury model, with increasing MVD of injured endometrium. MenSCs-CM could activate AKT and ERK pathways in HUVECs, induce the overexpression of eNOS, VEGFA, VEGFR1, VEGFR2 and Tie2, and promote cell proliferation, migration and angiogenesis. The activation of AKT and ERK is critical in angiogenesis induced by MenSCs-CM, and the angiogenesis induced by MenSCs-CM could be reversed by inhibitors of AKT and/or ERK. Taken together, our study indicates that MenSCs may play an active role in repairing the injured endometrium, which might be partially attributed to the involvement of angiogenesis mediated by MenSCs.

\section{Supplementary data}

This is linked to the online version of the paper at http://dx.doi. org/10.1530/REP-16-0286.

\section{Declaration of interest}

The authors declare that there is no conflict of interest that could be perceived as prejudicing the impartiality of the research reported.

\section{Funding}

This work was supported by the National Natural Science Foundation of China (grant numbers 81471505 and grant numbers 81270657), Zhejiang Medical science and Technology Project (grant numbers 2015RCA012 and 2016KYA110) and Zhejiang Science and Technology Project (grant number 2013C33236).

\section{References}

Alawadhi F, Du H, Cakmak H \& Taylor HS 2014 Bone marrow-derived stem cell (BMDSC) transplantation improves fertility in a murine model 
of Asherman's syndrome. PLOS ONE 9 e96662. (doi:10.1371/journal. pone.0096662)

Alcayaga-Miranda F, Cuenca J, Luz-Crawford P, Aguila-Diaz C, Fernandez A, Figueroa FE \& Khoury M 2015 Characterization of menstrual stem cells: angiogenic effect, migration and hematopoietic stem cell support in comparison with bone marrow mesenchymal stem cells. Stem Cell Research \& Therapy 6 32. (doi:10.1186/s13287-0150013-5)

Bockeria L, Bogin V, Bockeria O, Le T, Alekyan B, Woods EJ, Brown AA, Ichim TE \& Patel AN 2013 Endometrial regenerative cells for treatment of heart failure: a new stem cell enters the clinic. Journal of Translational Medicine 11 56. (doi:10.1186/1479-5876-11-56)

Boomsma RA \& Geenen DL 2012 Mesenchymal stem cells secrete multiple cytokines that promote angiogenesis and have contrasting effects on chemotaxis and apoptosis. PLOS ONE 7 e35685. (doi:10.1371/journal. pone.0035685)

Camussi G, Deregibus MC \& Tetta C 2010 Paracrine/endocrine mechanism of stem cells on kidney repair: role of microvesicle-mediated transfer of genetic information. Current Opinion in Nephrology and Hypertension 19 7-12. (doi:10.1097/MNH.0b013e328332fb6f)

Chen Y, Chang Y \& Yao S 2013 Role of angiogenesis in endometrial repair of patients with severe intrauterine adhesion. International Journal of Clinical and Experimental Pathology 6 1343-1350.

Dragneva G, Korpisalo P \& Yla-Herttuala S 2013 Promoting blood vessel growth in ischemic diseases: challenges in translating preclinical potential into clinical success. Disease Models \& Mechanisms 6 312-322. (doi:10.1242/dmm.010413)

Fang R, Cai L, Xiong F, Chen J, Yang W \& Zhao X 2016 The effect of endometrial thickness on the day of hCG administration on pregnancy outcome in the first fresh IVF/ICSI cycle. Gynecological Endocrinology 32 473-476. (doi:10.3109/09513590.2015.1132304)

Gaafar T, Osman O, Osman A, Attia W, Hamza H \& El Hawary R 2014 Gene expression profiling of endometrium versus bone marrowderived mesenchymal stem cells: upregulation of cytokine genes. Molecular and Cellular Biochemistry 395 29-43. (doi:10.1007/s11010014-2109-0)

Gargett CE \& Healy DL 2011 Generating receptive endometrium in Asherman's syndrome. Journal of Human Reproductive Sciences $\mathbf{4}$ $49-52$.

Hu X, Lin X, Jiang Y, Shi L, Wang J, Zhao L \& Zhang AS 2014 Establishment of mouse endometrial injury model by electrocoagulation. Zhonghua Yi Xue Za Zhi 94 3771-3773. (doi:10.3760/cma.j.is sn.0376-2491.2014.47.019)

Huang JJ, Shi YQ, Li RL, Hu A, Lu ZY, Weng L, Wang SQ, Han YP, Zhang L, Li B et al. 2015 Angiogenesis effect of therapeutic ultrasound on HUVECs through activation of the PI3K-Akt-eNOS signal pathway. American Journal of Translational Research 7 1106-1115.

Ichim TE, Alexandrescu DT, Solano F, Lara F, Campion Rde N, Paris E, Woods EJ, Murphy MP, Dasanu CA, Patel AN et al. 2010a Mesenchyma stem cells as anti-inflammatories: implications for treatment of Duchenne muscular dystrophy. Cellular Immunology 260 75-82. (doi:10.1016/j. cellimm.2009.10.006)

Ichim TE, Solano F, Lara F, Rodriguez JP, Cristea O, Minev B, Ramos F, Woods EJ, Murphy MP, Alexandrescu DT et al. 2010b Combination stem cell therapy for heart failure. International Archives of Medicine 35.

Jiang Z, Hu X, Yu H, Xu Y, Wang L, Chen H, Chen H, Wu R, Zhang Z, Xiang $\mathbf{C}$ et al. 2013 Human endometrial stem cells confer enhanced myocardial salvage and regeneration by paracrine mechanisms. Journal of Cellular and Molecular Medicine 17 1247-1260. (doi:10.1111/ jcmm.2013.17.issue-10)

Jing Z, Qiong Z, Yonggang W \& Yanping L 2014 Rat bone marrow mesenchymal stem cells improve regeneration of thin endometrium in rat. Fertility and Sterility 101 587-594. (doi:10.1016/j.fertnstert. 2013.10.053)

Kilic S, Yuksel B, Pinarli F, Albayrak A, Boztok B \& Delibasi T 2014 Effect of stem cell application on Asherman syndrome, an experimental rat model. Journal of Assisted Reproduction and Genetics 31 975-982. (doi:10.1007/s10815-014-0268-2)

Kinnaird T, Stabile E, Burnett MS, Lee CW, Barr S, Fuchs S \& Epstein SE 2004a Marrow-derived stromal cells express genes encoding a broad spectrum of arteriogenic cytokines and promote in vitro and in vivo arteriogenesis through paracrine mechanisms. Circulation Research 94 678-685. (doi:10.1161/01.res.0000118601.37875.ac)
Kinnaird T, Stabile E, Burnett MS, Shou M, Lee CW, Barr S, Fuchs S \& Epstein SE 2004b Local delivery of marrow-derived stromal cells augments collateral perfusion through paracrine mechanisms. Circulation 109 1543-1549. (doi:10.1161/01.cir.0000124062. 31102.57)

Kovacs P, Matyas S, Boda K \& Kaali SG 2003 The effect of endometrial thickness on IVF/ICSI outcome. Human Reproduction 18 2337-2341. (doi:10.1093/humrep/deg461)

Kwon HM, Hur SM, Park KY, Kim CK, Kim YM, Kim HS, Shin HC, Won MH, Ha KS, Kwon YG et al. 2014 Multiple paracrine factors secreted by mesenchymal stem cells contribute to angiogenesis. Vascular Pharmacology 63 19-28. (doi:10.1016/j.vph.2014.06.004)

Lai D, Wang F, Yao X, Zhang Q, Wu X \& Xiang C 2015 Human endometrial mesenchymal stem cells restore ovarian function through improving the renewal of germline stem cells in a mouse model of premature ovarian failure. Journal of Translational Medicine 13 155. (doi:10.1186/s12967015-0516-y)

Levens ED, Gustofson RL, Feinberg EC \& Larsen FW 2006 The ultrasonographic endometrial thickness and pattern of patients with fibroids is not significantly different than controls at the time of hCG administration in art cycles. Fertility and Sterility 86 S344-S345. (doi:10.1016/j.fertnstert.2006.07.936)

Lv M, Xia YF, Li B, Liu H, Pan JY, Li BB, Zhang C \& An FS 2016 Serum amyloid A stimulates vascular endothelial growth factor receptor 2 expression and angiogenesis. Journal of Physiology and Biochemistry 72 71-81. (doi:10.1007/s13105-015-0462-4)

Malinda KM 2003 In vivo matrigel migration and angiogenesis assays. Methods in Molecular Medicine 78 329-335.

March CM 2011 Management of Asherman's syndrome. Reproductive BioMedicine Online 23 63-76. (doi:10.1016/j.rbmo.2010.11.018)

Mou XZ, Lin J, Chen JY, Li YF, Wu XX, Xiang BY, Li CY, Ma JM \& Xiang C 2013 Menstrual blood-derived mesenchymal stem cells differentiate into functional hepatocyte-like cells. Journal of Zhejiang University Science B 14 961-972. (doi:10.1631/jzus.B1300081)

Murphy MP, Wang H, Patel AN, Kambhampati S, Angle N, Chan K, Marleau AM, Pyszniak A, Carrier E, Ichim TE et al. 2008 Allogeneic endometrial regenerative cells: an "Off the shelf solution" for critical limb ischemia? Journal of Translational Medicine 6 45. (doi:10.1186/14795876-6-45)

Nagori CB, Panchal SY \& Patel H 2011 Endometrial regeneration using autologous adult stem cells followed by conception by in vitro fertilization in a patient of severe Asherman's syndrome. Journal of Human Reproductive Sciences 4 43-48. (doi:10.4103/09741208.82360)

Patel AN, Park E, Kuzman M, Benetti F, Silva FJ \& Allickson JG 2008 Multipotent menstrual blood stromal stem cells: isolation, characterization, and differentiation. Cell Transplantation 17 303-311. (doi:10.3727/096368908784153922)

Rahbarghazi R, Nassiri SM, Khazraiinia P, Kajbafzadeh AM, Ahmadi SH, Mohammadi E, Molazem M \& Zamani-Ahmadmahmudi M 2013 Juxtacrine and paracrine interactions of rat marrow-derived mesenchymal stem cells, muscle-derived satellite cells, and neonatal cardiomyocytes with endothelial cells in angiogenesis dynamics. Stem Cells and Development 22 855-865. (doi:10.1089/ scd.2012.0377)

Romer T, Schmidt T \& Foth D 2000 Pre- and postoperative hormonal treatment in patients with hysteroscopic surgery. Contributions to Gynecology and Obstetrics 20 1-12. (doi:10.1159/000060283)

Schenker JG \& Margalioth EJ 1982 Intrauterine adhesions: an updated appraisal. Fertility and Sterility 37 593-610. (doi:10.1016/s00150282(16)46268-0)

Singh N, Mohanty S, Seth T, Shankar M, Bhaskaran S \& Dharmendra S 2014 Autologous stem cell transplantation in refractory Asherman's syndrome: a novel cell based therapy. Journal of Human Reproductive Sciences 7 93-98. (doi:10.4103/0974-1208.138864)

Thompson WD, Li WW \& Maragoudakis M 2000 The clinical manipulation of angiogenesis: pathology, side-effects, surprises, and opportunities with novel human therapies. Journal of Pathology 190 330-337. (doi:10.1002/(sici)1096-9896(200002)190:3<330::aid-path588> 3.0.co;2-4)

Togel F, Weiss K, Yang Y, Hu Z, Zhang P \& Westenfelder C 2007 Vasculotropic, paracrine actions of infused mesenchymal stem cells are important to the recovery from acute kidney injury. American Journal 
of Physiology: Renal Physiology 292 F1626-F1635. (doi:10.1152/ ajprenal.00339.2006)

Weidner N 1995 Current pathologic methods for measuring intratumoral microvessel density within breast carcinoma and other solid tumors. Breast Cancer Research and Treatment 36 169-180. (doi:10.1007) bf00666038)

Wu X, Luo Y, Chen J, Pan R, Xiang B, Du X, Xiang L, Shao J \& Xiang C 2014 Transplantation of human menstrual blood progenitor cells improves hyperglycemia by promoting endogenous progenitor differentiation in type 1 diabetic mice. Stem Cells and Development 23 1245-1257. (doi:10.1089/scd.2013.0390)

Yu D, Wong YM, Cheong Y, Xia E \& Li TC 2008 Asherman syndrome one century later. Fertility and Sterility 89 759-779. (doi:10.1016/j. fertnstert.2008.02.096)

Zeng Z, Huang WD, Gao Q, Su ML, Yang YF, Liu ZC \& Zhu BH 2015 Arnebin-1 promotes angiogenesis by inducing eNOS,
VEGF and HIF-1alpha expression through the PI3K-dependent pathway. International Journal of Molecular Medicine 36 685-697. (doi:10.3892/ijmm.2015.2292)

Zhong Z, Patel AN, Ichim TE, Riordan NH, Wang H, Min WP, Woods EJ, Reid M, Mansilla E, Marin GH et al. 2009 Feasibility investigation of allogeneic endometrial regenerative cells. Journal of Translational Medicine 7 15. (doi:10.1186/1479-5876-7-15)

Received 27 May 2016

First decision 30 June 2016

Revised manuscript received 13 July 2016

Accepted 1 August 2016 